Cerebrovascular Diseases 中文目录 2010 年第 30 卷 6 期

Table of Contents Vol.30, No. 6, 2010

翻译 马敏敏 审校刘新峰 徐格林（南京军区南京总医院神经内科, 南京大学医学院）

Translated by Minmin Ma and supervised by Xinfeng Liu, Gelin Xu, Dept. Neurology,

Nanjing Jinling Hospital, School of Medicine, Nanjing University

目前此翻译内容仅包括自 2004 年以来的《脑血管病》杂志目录。

For the moment, this translation service includes only the Table of Contents from

CEREBROVASCULAR DISEASES and starts with the first issue of the year 2004.

\title{
编者按 Editorial
}

5372010 年度本杂志专题论集--欧洲/日本卒中合作研究

Journal Anniversary Symposium 2010 Dedicated to Europe/Japan Stroke Research Cooperation

Hennerici, M.G. ; Minematsu, K.

Cerebrovasc Dis 2010;30:537-539

\section{论著 Original Paper}

540 重度颈内动脉狭窄和慢性脑损伤: 一项测定体积的磁共振影像研究

High-Grade Internal Carotid Artery Stenosis and Chronic Brain Damage: A Volumetric Magnetic

Resonance Imaging Study

Enzinger, C. ; Ropele, S. ; Gattringer, T. ; Langkammer, C. ; Schmidt, R. ; Fazekas, F.

Cerebrovasc Dis 2010;30:540-546

547 CT 灌注联合血管成像替代常规脑血管造影和乙酰唑胺激发的 SPECT 用于分流术后患者 随访

CT Perfusion with Angiography as a Substitute for both Conventional Digital Subtraction

Angiography and Acetazolamide-Challenged SPECT in the Follow-Up of Postbypass Patients

Park, J.C. ; Kim, J.E. ; Kang, H.-S. ; Sohn, C.-H. ; Lee, D.S. ; Oh, C.W. ; Han, M.H.

Cerebrovasc Dis 2010;30:547-555

556 缺血性卒中患者出院后的药物预防：年龄和性别的差异以及对死亡率的影响

Medical Prophylaxis following Hospitalization for Ischemic Stroke: Age- and Sex-Related

Differences and Relation to Mortality

Palnum, K.H. ; Mehnert, F. ; Andersen, G. ; Ingeman, A. ; Krog, B.R. ; Bartels, P.D. ; Johnsen, S.P.

Cerebrovasc Dis 2010;30:556-566

567 高龄和颈动脉内中膜增厚可预测颈动脉支架术相关的缺血事件

Older Age and Greater Carotid Intima-Media Thickness Predict Ischemic Events Associated with

Carotid-Artery Stenting

Rosenkranz, M. ; Thomalla, G. ; Havemeister, S. ; Wittkugel, O. ; Cheng, B. ; Krützelmann, A. ; Fie hler, J. ; Gerloff, C.

Cerebrovasc Dis 2010;30:567-572

573 偏侧空间忽略症的神经解剖基础: 一项基于体积像素分析的 SPECT 研究

Neural Correlates of Hemispatial Neglect: A Voxel-Based SPECT Study

Lee, B.H. ; Kang, E. ; Cho, S.S. ; Kim, E.-J. ; Seo, S.W. ; Kim, G.-M.; Lee, K.H.; Na,

D.L.

Cerebrovasc Dis 2010;30:573-583

584 急性卒中后癫㾁发作的预测因素 
Predictors of Acute Poststroke Seizures

Krakow, K. ; Sitzer, M. ; Rosenow, F. ; Steinmetz, H. ; Foerch, C.

Cerebrovasc Dis 2010;30:584-589

590 前瞻性巢式病例指涉研究显示血清胆红素和 UGT1A1*28 基因多态性不降低缺血性卒中 风险

Bilirubin and UGT1A1*28 Are Not Associated with Lower Risk for Ischemic Stroke in a

Prospective Nested Case-Referent Setting

Ekblom, K. ; Marklund, S.L. ; Johansson, L. ; Osterman, P. ; Hallmans, G. ; Weinehall, L. ; Wiklun

d, P.-G. ; Hultdin, J.

Cerebrovasc Dis 2010;30:590-596

597 短暂性脑缺血发作可误诊为显性局灶性蛛网膜下腔出血

Transient Ischaemic Attack Mimics Revealing Focal Subarachnoid Haemorrhage

Brunot, S. ; Osseby, G.V. ; Rouaud, O. ; Kazemi, A. ; Ricolfi, F. ; Couvreur, G. ; Catteau, A. ; Hervi eu, M. ; Moreau, T. ; Giroud, M. ; Béjot, Y.

Cerebrovasc Dis 2010;30:597-601

602 术中实施亚低温治疗的心脏手术患者发生卒中并发症的可能风险

The Possible Risk for Strokes Complicating Cardiac Surgery in Patients with Intraoperative

Hypothermia

Korn-Lubetzki, I. ; Steiner, I. ; Oren, A. ; Tauber, R. ; Steiner-Birmanns, B. ; Bitran, D.

Cerebrovasc Dis 2010;30:602-605

606 不稳定斑块的形态学分类及其与卒中早期复发的相关性

Morphological Classification of Mobile Plaques and Their Association with Early Recurrence of

Stroke

Ogata, T. ; Yasaka, M. ; Wakugawa, Y. ; Kitazono, T. ; Okada, Y.

Cerebrovasc Dis 2010;30:606-611

612 体外反搏可增加健康人的平均脑血流量吗? 体外反搏对健康人大脑中动脉血流速度和脑 血管调节反应的影响

Does External Counterpulsation Augment Mean Cerebral Blood Flow in the Healthy Brain? Effects of External Counterpulsation on Middle Cerebral Artery Flow Velocity and Cerebrovascular

Regulatory Response in Healthy Subjects

Jungehuelsing, G.J. ; Liman, T.G. ; Brunecker, P. ; Ebel, A. ; Endres, M. ; Buschmann, I. ; Pagonas, N. ; Buschmann, E.E.

Cerebrovasc Dis 2010;30:612-617

618 中国汉族人群中 MMP-3 功能基因多态性与 Moyamoya 病的相关性

Association of a Functional Polymorphism in the MMP-3 Gene with Moyamoya Disease in the Chinese Han Population

Li, H. ; Zhang, Z.-S. ; Liu, W. ; Yang, W.-Z. ; Dong, Z.-N. ; Ma, M. J. ; Han, C. ; Yang, H.; Cao,

W.-C.; Duan, L.

Cerebrovasc Dis 2010;30:618-625

卒中笔记 Stroke Note

626 伴有复视的急性眩晕一脑干卒中还是卒中假象?

Acute Vertigo with Double Vision - Brainstem Stroke or Stroke Mimic?

Schlaeger, R. ; Naegelin, Y. ; Welge-Lüssen, A. ; Straumann, D. ; Gass, A. ; Fluri, F. ; Baumann, T. Cerebrovasc Dis 2010;30:626-627 
附加区 Further Section

628 来自欧洲卒中组织的消息

ESO News

Cerebrovasc Dis 2010;30:628

629 作者索引

Author Index Vol.30, 2010

633 主题索引

Subject Index Vol.30,2010

目录

Contents Vol.30, 2010 